DOI: $10.17805 /$ zpu.2017.3.14

\title{
Возможности применения глобальных технологий Big Data для повышения эффективности логистических процессов *
}

\author{
О. Э. БАШИНА \\ МОСКОВСКИЙ ГУМАНИТАРНЫЙ УНИВЕРСИТЕТ, \\ Л. В. МАTPAEBA \\ РОССИЙСКИЙ ГОСУДАРСТВЕННЫЙ СОЦИАЛЬНЫЙ УНИВЕРСИТЕТ
}

На сегодняшний день информация расширяется с геометрической прогрессией за счет развития технологий в этой области и появления новых устройств. Однако обработка этих данных ставит все новые задачи перед исследователями, так как в подавляющем большинстве они являются неструктурированными и хаотичными. Это привело к появлению нового направления Big Data, в рамках которого формируются подходы к исследованию больших объемов данных для выявления шаблонов и раскрытия новой информации.

В рамках бизнес-процессов наиболее успешным и перспективным направлением является использование данных технологий в рамках логистистических процессов компаний. В связи с этим на основании эмпирического изучения актуального опыта зарубежных и российских компаний в данной области авторы систематизировали наиболее успешные, по их мнению, примеры, когда технологические нововведения в области Big Data позволили компаниям принципиально увеличить эффективность их деятельности. В ре-

* Исследование выполнено при финансовой поддержке РФФИ в рамках проекта проведения научных исследований «Разработка подходов к созданию системы оценки состояния и определения перспективных направлений развития научной сферы», проект № 16-02-00407, и в рамках проекта проведения научных исследований «Исследование потенциала отечественных производителей по обеспечению импортозамещения на потребительском рынке с использованием технологий Big Data», проект № 17-02-00718.

The research was financially supported by RFBS within the scientific research project "Development of Approaches to Creating a System Evaluating the State and Defining Perspective Directions of the Development of Scientific Sphere", project No. 16-02-00407, and within the scientific research project "Research of Domectic Manufacturers' Potential to Provide Import-Replacement on the Consumer Market using the Big Data Technologies", project No. 17-02-00718. 
зультате было выделено пять наиболее успешных направлений в области логистики: маршрутизация товаров и транспортных средств; планирование оперативной мощности компаний, анализ степени удовлетворенности клиентов, планирование бизнес-спроса. В рамках проведенного анализа авторы не только фиксируют появление новых специфических трендов и информационных решений в данной области, но и делают акцент на том, как использование определенных технологий в области Big Data способствовало повышению эффективности работы компании в целом.

Вместе с тем, несмотря на достижения в области технологических и методологических аспектов больших данных, существует большой неиспользованный потенциал в этой области для российских компаний, нераскрытие которого может привести к ослаблению их позиций уже в ближайшем будущем в высококонкурентных отраслях.

Ключевые слова: Big Data; методы сбора данных; методы обработки данных; статистика; логистические процессы

\section{ВВЕАЕНИЕ}

$\mathrm{C}$ егодняшняя «цифровая Вселенная» меняется и расширяется со скоростью, удваивающей объем данных каждые два года (Jeske, Grüner, Weiß, 2014). В дополнение к этому экспоненциальному росту объема информации существенно меняются и ее качественные характеристики.

Во-первых, расширяются типы самих данных за счет появления новых устройств, способных накапливать и передавать информацию: в нашу жизнь прочно вошли смартфоны, считыватели RFID, веб-камеры и сенсоры Сети. По сути, все эти устройства представляют собой огромное количество автономных источников данных, которые непрерывно генерируют потоки данных без вмешательства человека.

Во-вторых, подавляющее большинство вновь созданных данных происходит из изображений камеры видеонаблюдения, записи в блогах, форумов, дискуссий и каталогов электронной коммерции. Эти данные являются неструктурированными, хаотичными и не всегда достоверными. Все эти тенденции и привели к появлению принципиально нового направления - технологий обработки Big Data. B широком смысле слова - это совокупность подходов, инструментов и методов обработки как структурированных, так и неструктурированных данных огромных объемов массивов информации различного типа в условиях ее непрерывного прироста и актуализации (Матраева, Башина, 2017).

Наиболее эффективно, на наш взгляд, эти технологии сейчас реализуются в логистике, и наверное, не случайно, если вспомнить, что в переводе с древнегреческого «логистика» означает «искусство счета», т. е. логистика изначально являлась направлением, ориентированным на работу с данными. Сегодня логистические провайдеры управляют массовым потоком товаров, и в процессе этого управления, в свою очередь, создаются огромные наборы данных. И чем крупномасштабнее логистическая операция, тем выше требования к эффективной работе данных. Это понимают и сами логистические компании, что показали данные опроса «Тенденции и стратегии в области логистики», проведенного компанией BVL International (Handfield и др., 2013), по результатам которого $60 \%$ респондентов заявили, что планируют инвестировать в аналитику больших данных в течение следующих пяти лет.

На сегодняшний день пионерами и новаторами в области внедрения технологий Big Data являются глобальные и транснациональные компании, которые способствуют распространению достижений НТП в этой области (Сунаева, 2017; Ерохин, Матраева, Филатова, 2014). Именно поэтому изучение их опыта в этой сфере представляет несомненный интерес. 
Попытаемся систематизировать наиболее интересные тренды в области технологии Big Data по направлениям их применения в логистике.

ОПЫТ И НАПРАВАЕНИЯ ИСПОАБЗОВАНИЯ ТЕХНОАОГИЙ ВІG DАТА В ПРОЦЕССЕ МАРШРУТИЗАЦИИ ТОВАРОВ И ТРАНСПОРТНЫХ СРЕАСТВ

Безусловным лидером по количеству самых популярных решений в этой области является марирутизачия товаров и транспортных средств. Это связано с тем, что данные направления являются ключевыми факторами, влияющими на эффективность работы логистической системы любой компании, поскольку касаются не только времени доставки, расхода топлива, но даже и налогообложения, так как в некоторых странах оно связано с выбросами $\mathrm{CO}_{2}$ в атмосферу. Однако в настоящее время, как никогда раннее, авиакомпании, автотранспортные компании и железные дороги должны учитывать большое количество внешних факторов при решении этой задачи - понимать последствия изменения погодных условий, возможных пробок, ограничений времени работы транспорта, графиков технического обслуживания и множества других факторов.

Интересным примером при решении этой задачи является опыт компании DHL, реализующей подход, при котором используется ежедневное оптимизированное планирование доставок на основе динамической системы маршрутизации. Эта система пересчитывает маршруты в зависимости от текущих потребностей и дорожной ситуации, а также сокращает затраты и повышает эффективность использования $\mathrm{CO}_{2}$, например путем уменьшения пробега. При этом новым источником критической информации становятся датчики, установленные на транспортных средствах (Jeske, Grüner, Weiß, 2014).

Еще одним интересным, на наш взгляд, примером решения этой задачи является опыт компании UPS - одной из крупнейших американских международных логистических компаний. Эта компания применяет радиолокацию для отслеживания грузов, а затем собирает и анализирует показатели множества датчиков для контроля состояния транспортных средств и поведения водителей, использует данные мобильных CRM для мониторинга доставки и качества обслуживания клиентов. Аля оптимизации маршрутов и сокращения затрат в компании внедрена система ORION - одна из крупнейших в мире систем, основанных на результатах математической теории исследования операций. Аля решения задачи построения оптимального маршрута эта система использует картографические данные, данные о пунктах отправления и прибытия, размерах и требуемых сроках доставки грузов (12 кейсов по биг дате: ..., 2016).

\section{ИСПОАЬЗОВАНИЕ ТЕХНОАОГИЙ ВІG DАТА ПРИ ПААНИРОВАНИИ. ПААНИРОВАНИЕ ОПЕРАТИВНОЙ МОЩНОСТИ КОМПАНИЙ}

Вторым, но не менее перспективном, на наш взгляд, направлением является планирование оперативной мощности. Это направление включает оптимизацию планирования загрузочных мощностей для грузовых автомобилей, поездов и воздушных судов, а также планирование перераспределения персонала на складах. Часто при традиционном решении этой задачи используют исторические средние или личный опыт сотрудников, что нередко приводит к неэффективности принимаемых решений. Вместо этого, используя возможности расширенной аналитики, технологии Big Data позволяют оценить динамику не только внутри, но и вне распределительной сети. Моделируется и оценивается влияние на потребности в мощности на основании информации 
о доставке в режиме реального времени. Эти данные автоматически поступают из управления складом, систем и данных датчиков вдоль транспортной цепи. Кроме того, агрегируется информация о возможных изменениях спроса на основании внешних данных о клиентах. Например, данные о выпусках продуктов у конкурентов, новые открытия, которые могут повлиять на спрос, или информация о скрытом банкротстве. Аополнительно выявляются и классифицируются случайные инциденты (например, аварии или стихийные бедствия), которые могут повлиять на спрос. Точный прогноз на основе использовании технологии Big Data позволяет предугадывать сбои цепочки поставок и смягчать эффект непредвиденных инцидентов. Например, такие решения эффективно предсказывают предстоящие скопления на маршрутах или в пунктах транзита, которые не могут быть устранены компанией, но могут быть смягчены их последствия путем перемаршрутизации или ускорения других процессов.

В качестве примера можно привести опыт компании Union Pacific Railroad - крупнейшей железнодорожной компании США. На каждом составе компании были установлены термометры, акустические и визуальные сенсоры и другие датчики, данные которых аккумулируются в центр обработки. В этот центр также поступают данные о погодных условиях, состоянии тормозных систем, GPS-координаты составов. Разработанная модель позволяет отслеживать состояние колес и железнодорожного полотна и предсказывать сход составов с рельсов за несколько дней. Этого времени достаточно для того, чтобы оперативно устранить проблемы, избежать повреждений состава и задержки остальных поездов. В результате компании удалось снизить число схождений составов с рельсов на 75\% (12 кейсов по биг дате: ... , 2016).

\section{ИСПОАЬЗОВАНИЕ ТЕХНОАОГИИ ВІG DАТА АИЯ АНААИЗА СТЕПЕНИ УАОВАЕТВОРЕННОСТИ КАИЕНТОВ: \\ РАСШИРЕНИЕ СУЩЕСТВУЮЩИХ ГОРИЗОНТОВ}

Аогистические компании очень часто собирают отзывы клиентов, поскольку это обеспечивает не только понимание качества обслуживания, но и ожиданий, требований клиентов. Эта обратная связь является основным источником информации для непрерывного улучшения качества обслуживания, а также является источником новых идей в сфере поиска направлений возможных инноваций.

В прошлом единственным источником таких данных выступали система CRM (система управления взаимодействием с клиентом) и данные опросов клиентов. Но сегодня решения Big Data обеспечивают доступ к огромным объемам полезных данных, хранящихся на публичных интернет-сайтах. В социальных сетях и на форумах люди открыто и анонимно делятся опытом обслуживания и общения с компанией, но извлечение таких данных вручную - это поиск иголки в стоге сена. Аишь появление сложных методов обработки Big Data, таких как интеллектуальная обработка текста и семантическая аналитика, позволяют автоматически извлечь необходимую информацию из огромных хранилищ «текстов и звуков». И что еще является неоспоримым достоинством, так это тот факт, что незапрашиваемая постоянная обратная связь может быть структурирована по различным направлениям: регион, время, пол и т. А.

Примером может является созданная на основе методов обработки Big Data прогнозная модель поведения клиентов на основании изучения социальных отношения (связей) между ними, достаточно популярная уже не только в логистическом бизнесе. Эта модель, а точнее сказать - уже целый класс подобных моделей, при анализе структуры социальной сети стремится выделить однородные группы («племя») с вы- 
раженными лидерами (люди, которые оказывают сильное влияние в больших, связанных группах). Модель исходит из постулата, что, если лидер переключается к услуге или продукту конкурента, вполне вероятно, что друзья, члены семьи или подписчики в социальных сетях также переключатся на этот продукт или услугу.

\section{ПРИМЕР ИСПОАЬЗОВАНИЯ ВІG DАТА АИЯ УПРАВАЕНИЯ РИСКАМИ ПОСТАВОК И ПРОИЗВОАИТЕАЬНОСТЬЮ ПОСТАВЩИКОВ}

Примером оригинального решения данной задачи может являться опыт компании GE Oil \& Gas, занимающейся производством высокотехнологичного оборудования Аля нефтегазового сектора. В условиях снижения цен на энергоресурсы цена простоя оборудования из-за поломок может обойтись добывающим компаниям очень дорого. Понимая этот факт, компания решила установить на продаваемом оборудовании датчики, которые передают оперативную информацию о состоянии нефтедобычи, а собственно для анализа этих данных была разработана облачная платформа Predix, которая, используя алгоритмы машинного обучения, позволяла инженерам составлять расписания диагностических проверок и снижать время «простоя», выявляя возможные неисправности до того, как они произойдут (Семь кейсов использования ..., 2017).

\section{АИАЕММА ТОЧНОГО ПААНИРОВАНИЯ БИЗНЕС-СПРОСА И ЕЕ РЕШЕНИЕ С ПОМОЩЬЮ ВIG DАТА}

Это достаточно распространенная дилемма в логистике практически любого преАприятия: отсутствие продукта на складе может привести к отказу клиента от компании, а затоваривание склада - к финансовым убыткам.

Примером может является решение компании Otto Group (крупнейшей многоканальной розничной компании, торгующей одеждой по каталогам). Она осознала, что традиционные методы прогнозирования спроса оказались неадекватными в условиях все более повышающейся конкуренции. Оценив ряд решений для создания стабильных прогнозов объемов продаж, Otto Group в конечном итоге использовала метод прогнозирования, возникший в области физики высоких энергий. Этот метод базируется на многомерном анализе и использует инструментарий, возможности самообучения от нейронных сетей и объединяет их с байесовской статистикой 1 . C помощью этого инструмента был разработан новый алгоритм прогнозирования: он был обучен на основании исторических данных 16 предыдущих лет и имел непрерывные входы с 300 миллионами записей транзакций в неделю от текущего года. Эта новая система генерирует более одного миллиарда индивидуальных прогнозов на каждый год и уже дала убедительные результаты. Только 11\% әлементов каталога ОТТО имеют отклонения по данному прогнозу более чем на 20\% (Sinn, 2012).

\section{ЗАКАЮЧЕНИЕ}

В заключение хотелось бы отметить, что на практике существуют многочисленные препятствия в области реализации решений в Big Data. B первую очередь, к ним можно отнести низкое качество данных, конфиденциальность некоторых из них и сложную техническую осуществимость. Но, на наш взгляд, в долгосрочной перспективе эти препятствия отойдут на второй план, поскольку перед глазами всегда будет именно опыт их успешного применения, который превратил Google, Amazon, Facebook и еВау в лидеров информационного рынка. Сейчас прорывные решения в области Big 
Data сосредоточены именно в логистике, так как там в них возникла наибольшая потребность. Аостижения в области технологических и методологических аспектов больших данных обеспечивают большие преимущества в секторе логистики. Существует большой неиспользованный потенциал для улучшения операционной эффективности, приобретения опыта работы с клиентами и создания новых бизнес-моделей. Эта статья иллюстрирует потенциал решений в логистической сфере и доказывает, что Big Data уже стала движущей конкурентной силой в высококонкурентных отраслях, но помимо технологических изменений неизбежным спутником әффективного внедрения Big Data должны быть и организационные изменения.

\section{ПРИМЕЧАНИЕ}

1 Здесь - статистическая модель, предполагающая обновление сложившихся представлений в свете полученного опыта.

\section{СПИСОК АИТЕРАТУРЫ}

12 кейсов по биг дате: подтвержденные примеры из индустрии, когда биг дата приносит деньги (2016) [Электронный ресурс] // Хабрахабр. 10 ноября. URL: https://habrahabr.ru/company/npl/blog/314926/ (дата обращения: 24.05.2017).

Ерохин, С. Г., Матраева, А. В., Филатова, Ю. М. (2014) Иностранные инвестиции. М. : Аашков и К. 248 с.

Измалкова, С. А., Головина, Т. А. (2015) Использование глобальных технологий «ВIG DATA» в управлении экономическими системами // Известия Тульского государственного университета. Экономические и юридические науки. № 4-1. С. 151-158.

Матраева, А. В., Башина, О. Э. (2017) Современные тренды использования технологии big data в экономических процессах в практике зарубежных и отечественных компаний // Экономика и предпринимательство. № 5-1 (82-1). С. 788-791.

Семь кейсов использования технологий Big Data в сфере производства [Электронный ресурс]// Хабрахабр. 3 апреля. URL: https://habrahabr.ru/company/npl/blog/325550/ (дата обращения: 24.05.2017).

Сунаева, Ю. В. (2017) Последствия транснационализации для национальной экономики // Актуальные вопросы развития мировой и модернизации российской экономики. Курск : Университетская книга. С. 88-93.

Шавшина, С. А. (2015) Возможности использования больших данных в российском госсекторе // Экономика и предпринимательство. №9-1 (62-1). С. 321-326.

Handfield, R., Straube, F., Pfohl, H., Wieland, An. (2013) Trends and Strategies in Logistics and Supply Chain Management. BVL International. 81 p.

Jeske, M., Grüner, M., Weiß, F. (2014) Big Data in Logistics. DHL. 31 p.

Sinn, M. (2012) Big Data \& Predictive Analytics / Otto Group// Conference Talk "Big Data Europe". Zurich. August 28. 28 p.

Lата поступления: 03.07.2017 2.

\section{POSSIBILITIES OF APPLICATION OF THE GLOBAL BIG DATA TECHNOLOGIES FOR INCREASING THE EFFICIENCY OF LOGISTICS PROCESSES \\ O. E. BASHINA \\ MOSCOW UNIVERSITY FOR THE HUMANITIES, \\ L. V. MATRAEVA \\ RUSSIAN STATE SOCIAL UNIVERSITY}

Nowadays, information is expanding with geometric progression due to the development of technologies in this area and the emergence of new devices. However, the processing of these data poses 
new challenges for researchers, since in the overwhelming majority those data are unstructured and chaotic. This led to the emergence of a new direction of Big Data, within which approaches to the study of large amounts of data are formed to identify patterns and to disclose new information.

Within the framework of business processes, the most successful and promising direction is the use of these technologies within the framework of logistic processes of companies. In this connection, on the basis of the empirical study of the actual experience of foreign and Russian companies in this field, the authors systematized the most successful examples in their opinion when technological innovations in the field of Big Data allowed companies to increase their activity efficiency in principle. As a result, five most successful areas in the field of logistics were identified: routing of goods and vehicles; planning of the operational capacity of companies, analysis of the degree of customer satisfaction, planning of business demand. In the framework of the conducted analysis, the authors not only record the emergence of new specific trends and information solutions in this area, but also emphasize how the use of certain technologies in the field of Big Data contributed to improving the efficiency of the company as a whole.

At the same time, despite the achievements in the field of technological and methodological aspects of large data, there is a great untapped potential in this area for Russian companies, whose non-disclosure may lead to the weakening of their positions in the near future in highly competitive industries.

Keywords: Big Data; methods of data collection; methods of data processing; statistics; logistics processes

\section{REFERENCES}

12 keisov po big date: podtverzhdennye primery iz industrii, kogda big data prinosit den'gi (2016). Khabrakbabr, 10 November [online] Available at: https://habrahabr.ru/company/npl/blog/314926/ (access date: 24.05.2017). (In Russ.).

Erokhin, S. G., Matraeva, L. V. and Filatova, Iu. M. (2014) Inostrannye investitsii. Moscow, "Dashkov i K" Publ. 248 p. (In Russ.).

Izmalkova, S. A. and Golovina, T. A. (2015) Ispol'zovanie global'nykh tekhnologii «BIG DATA» $\mathrm{v}$ upravlenii ekonomicheskimi sistemami. Izvestiia Tul'skogo gosudarstvennogo universiteta. Ekonomicheskie i iuridicheskie nauki, no. 4-1, pp. 151-158. (In Russ.).

Matraeva, L. V. and Bashina, O. E. (2017) Sovremennye trendy ispol'zovaniia tekhnologii big data $\mathrm{v}$ ekonomicheskikh protsessakh v praktike zarubezhnykh i otechestvennykh kompanii. Ekonomika i predprinimatel'stvo, no. 5-1 (82-1), pp. 788-791. (In Russ.).

Sem' keisov ispol'zovaniia tekhnologii Big Data v sfere proizvodstva. Khabrakbabr, 3 April [online] Available at: https://habrahabr.ru/company/npl/blog/325550/ (access date: 24.05.2017). (In Russ.).

Sunaeva, Iu. V. (2017) Posledstviia transnatsionalizatsii dlia natsional'noi ekonomiki. In: Aktual' nye voprosy razvitiia mirovoi i modernizatsii rossiiskoi ekonomiki. Kursk, Universitetskaia kniga. Pp. 88-93. (In Russ.).

Shavshina, S. A. (2015) Vozmozhnosti ispol'zovaniia bol'shikh dannykh v rossiiskom gossektore. Ekonomika i predprinimatel' stvo, no. 9-1 (62-1), pp. 321-326.

Handfield, R., Straube, F., Pfohl, H. and Wieland, An. (2013) Trends and Strategies in Logistics and Supply Chain Management. BVL International. $81 \mathrm{p}$.

Jeske, M., Grüner, M. and Weiß, F. (2014) Big Data in Logistics. DHL. 31 p.

Sinn, M. (2012) Big Data \& Predictive Analytics / Otto Group. In: Conference Talk "Big Data Europe". Zurich. 28 p.

Submission date: 03.07.2017.

Башина Ольга Эмильевна - доктор экономических наук, профессор, заведующая кафедрой статистики, маркетинга и бухгалтерского учета Московского гуманитарного университета. Адрес: 111395, Россия, г. Москва, ул. Юности, А. 5. Тел.: +7 (499) 374-70-20. Эл. адрес: bashina_о_ e@mail.ru 
Матраева Иилия Валериевна - доктор экономических наук, профессор кафедры экономической теории и мировой экономики Российского государственного социального университета. Адрес: 129226, Россия, г. Москва, ул. Вильгельма Пика, д. 4, стр. 1. Тел.: +7 (495) 255-67-67, доб. 2079. Эл. алрес: matraeva@rambler.ru

Bashina Olga Emilievna, Doctor of Economics, Professor; Head, Department of Statistics, Marketing and Accounting, Moscow University for the Humanities. Postal address: 5, Yunosti St., Moscow, Russian Federation 111395. Tel.: +7 (499) 374-70-20. E-mail: bashina_o_e@mail.ru

Matraeva Liliya Valerievna, Doctor of Economics, Professor, Department of Economic Theory and World Economy, Russian State Social University. Postal address: 4, Bldg. 1, Vilgelma Pika St., Moscow, Russian Federation 129226. Tel.: +7 (495) 255-67-67, ext. 2079. E-mail: matraeva@rambler.ru 